\title{
Measurement of local composition of organic materials within porous inorganic media using two-dimensional acoustic mapping
}

\author{
M. Cassiede, and J.M. Shaw \\ Department of Chemical and Materials Engineering, University of Alberta, Edmonton, Canada
}

Real-time measurement of local composition and movement of organic fluids in porous media presents numerous data acquisition and data processing challenges in many fields of science. Imaging techniques based on Xrays, acoustic or thermo-acoustic devices have been used to study the evolution of the structure of cells and internal organs in biology and biomedical applications or to monitor thermophysical phenomena such as diffusion, catalysis, phase change, and natural gas hydrate formation in chemical engineering. Acoustic techniques are of growing interest as they are often more convenient, cheaper, and have fewer side effects in the case of biomedical applications. While the data acquisition time required to obtain high-resolution three-dimensional images can be long, current commercial multi-element acoustic devices permit the generation of two-dimensional high-resolution acoustic images from ultrasonic emission from an array of sensors at short time intervals. In principle, it is possible to monitor a physical property as a function of time and at small length scales. In this work, a micro seismic experimental technique is illustrated, that provides realtime, two-dimensional, high-resolution images of fluid flow and fluid composition within inorganic porous media.

More specifically, two 64 element acoustic probes 10L64-A2 purchased from Olympus NDT were placed on opposite sides of porous media of interest and operated in sender/receiver (transmission) mode [1]. Acoustic beams were generated by exciting groups of elements simultaneously or with time delays. In this way, beams traversed the porous medium over a range of angles from $40^{\circ}$ to $40^{\circ}$. Data acquisition hardware (TomoScan Focus LT) and software (TomoView Software) from Olympus NDT were used for data acquisition. Two-dimensional acoustic maps were generated at $60 \mathrm{~s}$ intervals based on time of flight data captured for a thousand transmitterreceiver pairs with a spatial resolution of $50 \mu \mathrm{m}$ using an inversion LSQR algorithm [2].

Automation, validation and optimization of the data acquisition procedure have proved to be significant tasks and are key themes in the technique development to date. Figure 1 comprises an illustrative example showing a scaled drawing and a corresponding acoustic map for three precisely machined aluminum cylinders imbedded in a polyvinyl chloride polymer matrix. Cylinder centroids are well placed and the vertical dimension is well identified. There is some residual size distortion horizontally. Preliminary acoustic map sequences illustrating transport phenomena in porous media are also presented. Examples include imbibition and diffusion of water and hydrocarbons in packed beds of 200-500 $\mu \mathrm{m}$ diameter silica particles, and forced convective mass transfer at a fluid/natural porous medium interface (e.g.: limestone or coal).
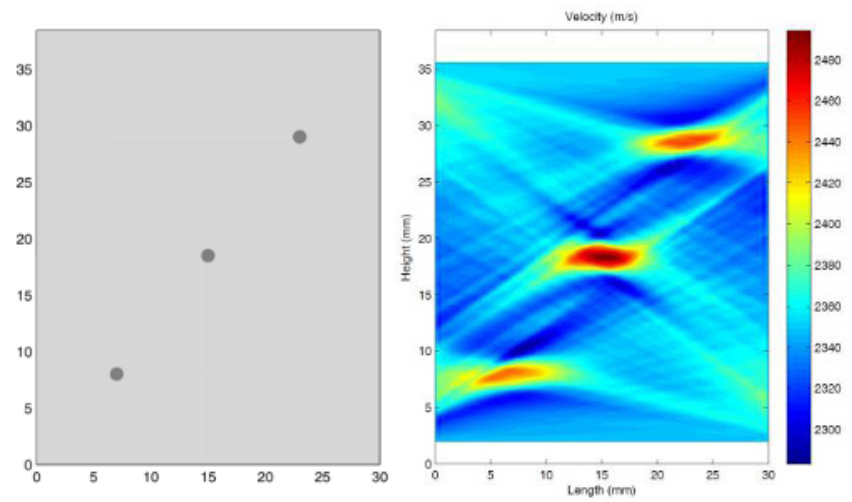

Figure 1. $30 \times 40 \mathrm{~mm}$ PVC plate with three aluminum cylinders (1.2 mm diameter): (a) drawing, (b) preliminary acoustic map.

\section{References}

1. M. Khammar, J.M. Shaw, Review of Scientific Instruments 82104902 (2011)

2. B. Giroux, E. Gloaguen, Computers and Geosciences $\mathbf{3 3}$ 126 (2007) 